\title{
A novel intracerebral hemorrhage-induced rat model of neurogenic voiding dysfunction: Analysis of lower urinary tract function
}

\author{
YOUNG-SAM CHO ${ }^{1}$, IL-GYU KO ${ }^{2}$, CHANG-JU KIM ${ }^{2}$ and KHAE-HAWN KIM ${ }^{3}$ \\ ${ }^{1}$ Department of Urology, Kangbuk Samsung Hospital, Sungkyunkwan University School of Medicine, Seoul 110-746; \\ ${ }^{2}$ Department of Physiology, Kyung Hee University College of Medicine, Seoul 130-701; ${ }^{3}$ Department of Urology, \\ Gachon University School of Medicine, Gil Medical Center, Incheon 405-760, Republic of Korea
}

Received December 12, 2013; Accepted September 18, 2014

DOI: $10.3892 / \mathrm{mmr} .2015 .3720$

\begin{abstract}
Neurogenic lower urinary tract dysfunction (NLUTD) is a major problem in patients with various neurological disorders, and may result in debilitating symptoms and serious complications, including chronic renal failure and recurrent urinary tract infections. Clinically, stroke is associated with voiding dysfunction. However, lower urinary tract function evaluation in an intracerebral hemorrhage (ICH) model has not, to the best of our knowledge, been reported Therefore, in the present study, lower urinary tract function in $\mathrm{ICH}$-induced rats was investigated and the results were compared with those obtained in normal rats. The effects of ICH on peripheral bladder function and central micturition centers [medial preoptic area, ventrolateral gray, pontaine micturition center and spinal cord (lumbar 4 (L4)-L5)] were also examined. Adult female Sprague-Dawley rats were divided into two groups: Control $\mathrm{ICH}$-induced. Induction of $\mathrm{ICH}$ in the hippocampal CA1 region was performed using a stereotaxic frame and type IV collagenase. The effects of ICH on the central micturition centers were investigated by simultaneously determining the extent of neuronal activation (c-Fos) and nerve growth factor (NGF) expression, and assessing voiding function (urodynamically using cystometry). The results revealed that induction of ICH significantly enhanced bladder contraction pressure and time, while simultaneously reducing voiding pressure and time. Furthermore, the c-Fos and NGF expression levels in the neuronal voiding centers were significantly increased in the rats with induced ICH as compared with the control rats. Therefore, this $\mathrm{ICH}$-induced
\end{abstract}

Correspondence to: Professor Khae-Hawn Kim, Department of Urology, Gachon University School of Medicine, Gil Medical Center, 21 Namdong-daero, 774 Beon-gil, Namdong-gu, Incheon 405-760, Republic of Korea

E-mail: kimcho99@gilhospital.com

Key words: cerebral hemorrhage, neurogenic urinary bladder, urodynamics, animal models, Fos, nerve growth factor
NLUTD rat model may be a more appropriate method to analyze NLUTD in stroke patients than a cerebral infarction model, as the former more accurately reflects the nature of the hemorrhage in the two types of stroke.

\section{Introduction}

Neurogenic lower urinary tract dysfunction (NLUTD) is a major problem in patients with various neurological disorders, and may result in debilitating symptoms and serious complications, including chronic renal failure and recurrent urinary tract infections. Important causes of NLUTD include cerebrovascular accident, Parkinson's disease, spinal cord injury (SCI), multiple sclerosis, diabetes mellitus and peripheral nerve injury due to radical abdominal or pelvic surgery. NLUTD is also common in stroke patients, and the evaluation of bladder function and management of this disorder should be recognized as part of routine rehabilitation (1-3). Occasionally, successful treatment is difficult to quantify due to the irreversibility or progressive nature of strokes. Therefore, neurological investigation of NLUTD in stroke patients is urgently required.

The majority of animal experimental studies of NLUTD have been directed towards the development of models of overactive bladder (OAB) (4). Various animal models of OAB have been analyzed, including a spontaneous hypertensive rat model and a rat model of dopaminergic brain lesions, and various methods have been used to induce OAB, including autoimmune encephalomyelitis, experimental fluid percussion injury to the brain and cerebral infarction using ligation of the common carotid arteries (4). SCI animal models generated by spinal cord compression using electromagnetically controlled watch-maker forceps or spinal cord transection using iridectomy microscissors have also been used to investigate OAB $(5,6)$. Furthermore, bladder outlet obstruction (BOO) models to examine $\mathrm{OAB}$ have been generated using a small polyethylene catheter to produce partial urethral obstruction or by intraperitoneal (i.p.) cyclophosphamide (CYP) injection, due to the ease and simplicity of these techniques $(7,8)$. However, these models may not adequately address the characteristics of hemorrhage in stroke patients, although cerebral infarction models are appropriate for ischemic stroke studies. 
In addition, the relevance of observed changes following SCI, BOO and CYP injection may not reflect conditions in human stroke patients in terms of the possible pathophysiology of NLUTD.

An intracerebral hemorrhage (ICH) model obtained by direct collagenase injection into the brain has been used in numerous neurological studies to evaluate the pathophysiological changes in the brain following ICH and to determine the therapeutic options for treating $\operatorname{ICH}(9,10)$. To the best of our knowledge, in the urological literature, no studies that use an ICH model to analyze NLUTD, including OAB and lower urinary tract function, have been reported. Thus, in the present study, lower urinary tract function in $\mathrm{ICH}$-induced rats was investigated and compared with that in normal rats. The effects of ICH on NLUTD with regard to peripheral bladder function and central micturition centers [medial preoptic area (MPA), ventrolateral periaqueductal gray (vlPAG), pontaine micturition center (PMC) and spinal cord (lumbar 4 (L4)-L5)] were also examined.

\section{Materials and methods}

Experimental animals and treatments. Adult female Sprague-Dawley rats, weighing $260 \pm 10 \mathrm{~g}$ and aged 10 weeks, were obtained from a commercial breeder (Orient Co., Seoul, Korea). The present study was performed in accordance with the animal care guidelines of the National Institutes of Health and the Korean Academy of Medical Sciences (Seoul, Korea), and was approved by the Kyung Hee University Institutional Animal Care and Use Committee (Seoul, Korea). Each animal was housed under controlled temperature $\left(23 \pm 2^{\circ} \mathrm{C}\right.$ ) and lighting (8:00 a.m.-8:00 p.m.) conditions with food and water available ad libitum prior and subsequent to experiments. The animals were randomly divided into the following two groups ( $n=10$ in each group): Control and ICH-induced.

ICH induction. To induce $\mathrm{ICH}$, the rats were anesthetized with Zoletil ${ }^{\circledR} 50$ (10 mg/kg, i.p.; Vibac Laboratories, Carros, France) and placed in a stereotaxic frame. The needle of a $10 \mu 1$ Hamilton syringe (Micro 701; Hamilton company, Reno, $\mathrm{NV}$, USA) was inserted through a burr hole into the right hippocampus to the following coordinates: $2.2 \mathrm{~mm}$ anterior, $2.2 \mathrm{~mm}$ lateral and $4.2 \mathrm{~mm}$ depth to bregma. A solution (1 $\mu \mathrm{l}$ ) containing $0.2 \mathrm{U}$ collagenase (Type IV; Sigma-Aldrich, St. Louis, MO, USA) was infused over $1 \mathrm{~min}$. The needle was maintained in place for an additional 3 min following the infusion and was subsequently withdrawn slowly. The animals in the sham-operation group received $1 \mu \mathrm{l}$ physiological saline by the same method.

Cystometry. Rat bladder function was evaluated by cystometry 14 days after the ICH surgery. The rats were anesthetized with $10 \mathrm{mg} / \mathrm{kg}$ Zoletil@ 50 (i.p.). A sterile polyethylene catheter (PE50) was inserted into the urethra through the bladder dome. The catheter was connected to a pressure transducer (Harvard Apparatus, Holliston, MA, USA) and syringe pump (Harvard Apparatus) via a three-way stopcock to record the intravesical pressure. When the bladder had emptied, cystometry was conducted by infusing $0.5 \mathrm{ml}$ saline into the bladder.
Table I. Changes in urodynamic parameters, c-Fos and NGF expression following the induction of ICH.

\begin{tabular}{|c|c|c|}
\hline Variable & Control & $\mathrm{ICH}$ \\
\hline \multicolumn{3}{|l|}{$\begin{array}{l}\text { Bladder contraction } \\
\text { parameter }\end{array}$} \\
\hline Pressure $\left(\mathrm{cm} \mathrm{H}_{2} \mathrm{O}\right)$ & $3.30 \pm 0.13$ & $11.97 \pm 0.64^{\mathrm{a}}$ \\
\hline Time (sec) & $16.58 \pm 0.50$ & $19.89 \pm 0.70^{\mathrm{a}}$ \\
\hline \multicolumn{3}{|l|}{ Voiding parameter } \\
\hline Pressure $\left(\mathrm{cm} \mathrm{H}_{2} \mathrm{O}\right)$ & $38.23 \pm 1.86$ & $29.93 \pm 1.50^{\mathrm{a}}$ \\
\hline Time (sec) & $82.00 \pm 15.90$ & $55.78 \pm 10.00^{\circ}$ \\
\hline \multicolumn{3}{|l|}{$\begin{array}{l}\text { c-Fos expression } \\
\text { (no. cells/section) }\end{array}$} \\
\hline MPA & $33.22 \pm 3.11$ & $87.20 \pm 5.19^{a}$ \\
\hline vlPAG & $39.50 \pm 2.88$ & $81.20 \pm 5.69^{a}$ \\
\hline PMC & $22.44 \pm 3.09$ & $70.10 \pm 4.01^{\mathrm{a}}$ \\
\hline L4-L5 & $13.55 \pm 1.80$ & $41.09 \pm 4.11^{\mathrm{a}}$ \\
\hline \multicolumn{3}{|l|}{$\begin{array}{l}\text { NGF expression } \\
\text { (no. cells/section) }\end{array}$} \\
\hline MPA & $46.38 \pm 9.01$ & $99.44 \pm 10.19$ \\
\hline vlPAG & $39.14 \pm 6.90$ & $104.45 \pm 11.39^{\circ}$ \\
\hline PMC & $30.61 \pm 5.10$ & $79.50 \pm 7.20^{\mathrm{a}}$ \\
\hline L4-L5 & $21.45 \pm 3.10$ & $56.11 \pm 5.10^{\mathrm{a}}$ \\
\hline
\end{tabular}

Data are presented as the mean \pm standard error of the mean. $\mathrm{N}=10$ in each group. ${ }^{\text {a }}<0.05$ vs. the control group. NGF, nerve growth factor; ICH, intracerebral hemorrhage; MPA, medial preoptic area; vlPAG, ventrolateral periaqueductal gray; $\mathrm{PMC}$, pontaine micturition center; L, lumbar spinal cord.

Bladder contraction pressure and time were monitored using Labscribe (iWork System Inc., Dover, NH, USA).

Preparation of tissues. The rats were sacrificed immediately following cystometric determination of the contraction pressure and time in the bladder. Briefly, the animals were anesthetized with Zoletil $50^{\circledR}$ (10 mg/kg, i.p.; Vibac Laboratories), and were transcardially perfused with $50 \mu \mathrm{m}$ phosphate-buffered saline (PBS), followed by $4 \%$ paraformaldehyde in $100 \mathrm{~mm}$ sodium phosphate buffer at $\mathrm{pH}$ 7.4. The brain was removed, postfixed in the same fixative overnight and transferred to a $30 \%$ sucrose solution for cryoprotection. Subsequently, $40 \mu \mathrm{m}$ serial coronal sections were produced with a freezing microtome (Leica Biosystems Nussloch GmbH, Nussloch, Germany). The PMC was defined as the region spanning Bregma -9.68 to $-9.80 \mathrm{~mm}$, the vlPAG as the region spanning Bregma -7.64 to $-8.00 \mathrm{~mm}$, the MPA as the region spanning Bregma -0.26 to $0.80 \mathrm{~mm}$ and the spinal cord as the L4-L5 region. An average of 10 sections were collected from each rat for each region and were used for immunohistochemical analysis.

Immunohistochemistry for c-Fos and nerve growth factor $(N G F)$. Rabbit polyclonal anti-c-Fos (cat. no. sc-52) or mouse monoclonal anti-NGF (cat. no. sc-365944) antibodies (Santa 
A
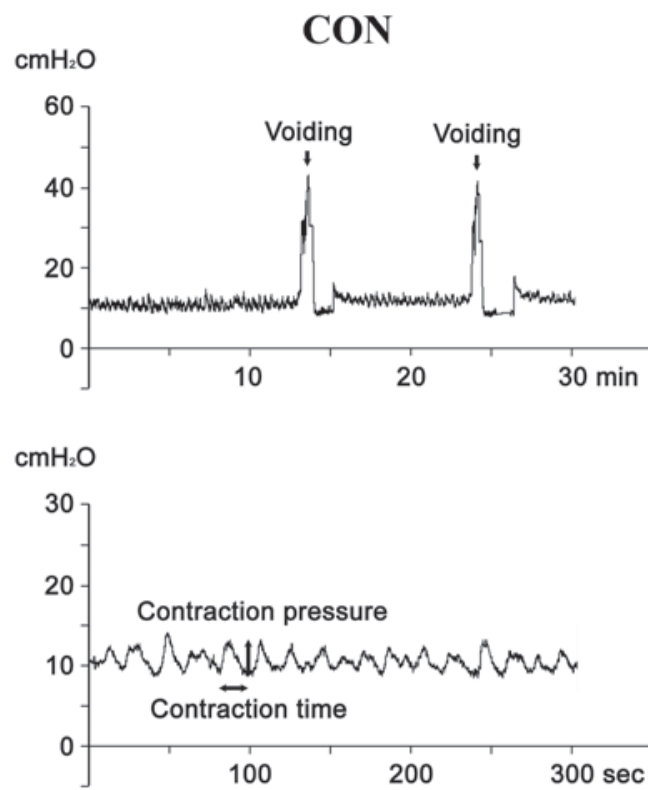

B
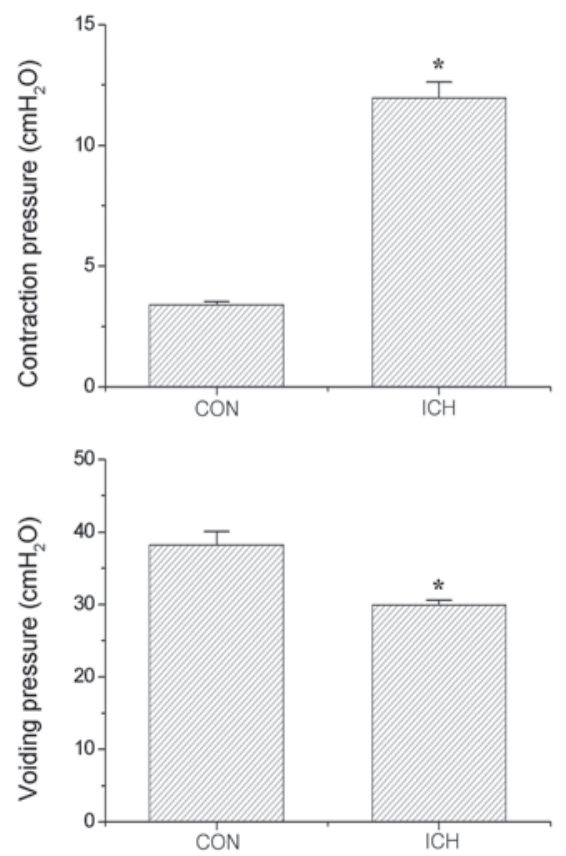

ICH
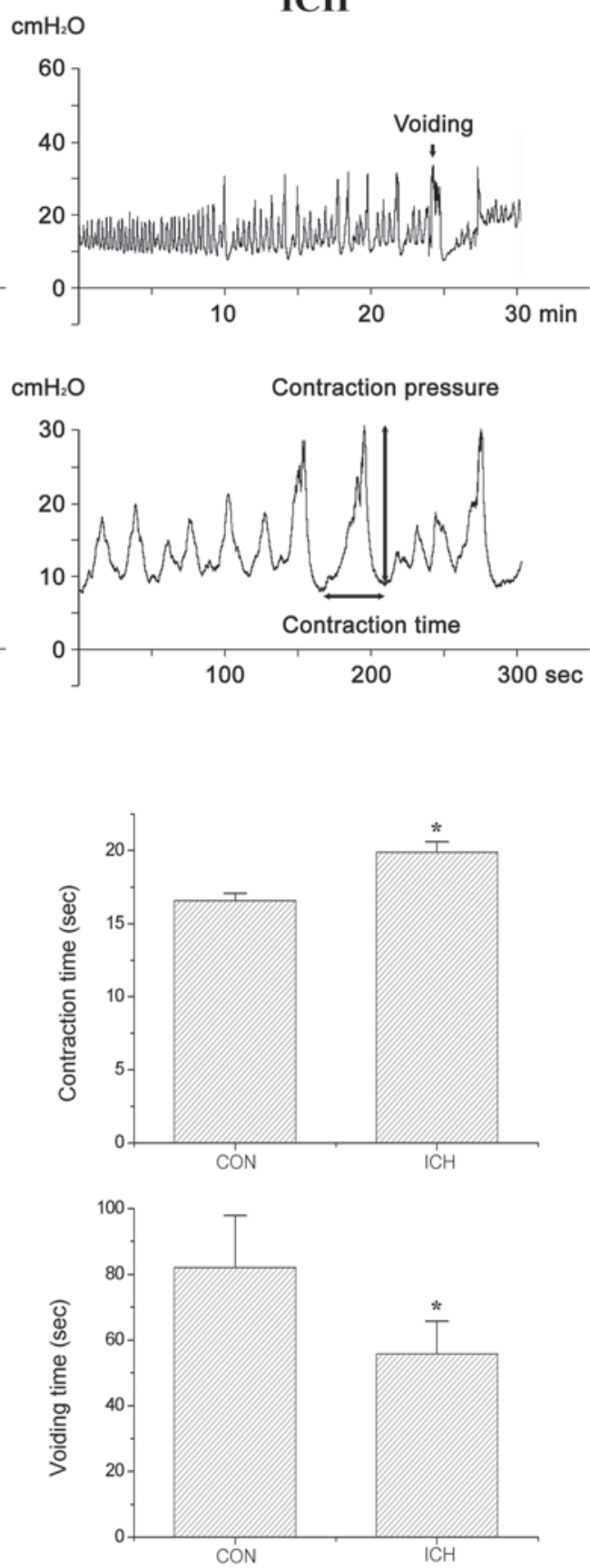

Figure 1. Effect of induction of ICH on urodynamic parameters. (A) Cystometry results for each group. (B) Analysis of bladder contraction (pressure/time) and voiding function (pressure/time). Results are presented as the mean \pm standard error of the mean. ${ }^{*} \mathrm{P}<0.05$ vs. the control group. ICH, intracerebral hemorrhage; CON, control group.

Cruz Biotechnology, Inc., Santa Cruz, CA, USA) at 1:1,000 dilutions were incubated overnight with free-floating tissue sections at $4^{\circ} \mathrm{C}$. The sections were washed three times with PBS for $3 \mathrm{~min}$, and were subsequently incubated for $1 \mathrm{~h}$ at room temperature with biotinylated anti-rabbit (cat. no. BA1000) or anti-mouse (cat. no. BA2000) secondary antibodies (1:200 dilution; Vector Laboratories, Burlingame, CA, USA) as appropriate. After washing three times with PBS for $3 \mathrm{~min}$, the sections were incubated for $1 \mathrm{~h}$ at room temperature with avidin-biotin-peroxidase complex (Vector Laboratories). The sections were washed a further three times with PBS, and were then incubated in a solution consisting of
$0.05 \%$ 3,3'-diaminobenzidine (Sigma-Aldrich) and 0.01\%

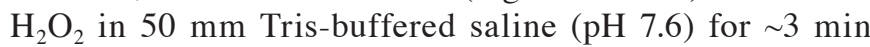
to visualize immunoreactivity. The sections were mounted onto gelatine-coated slides subsequent to washing three times with PBS. The slides were air-dried at room temperature overnight and coverslips were mounted using Permount ${ }^{\mathrm{TM}}$ Mounting Medium (Fisher Scientific, Waltham, MA, USA).

Data analysis and statistics. The numbers of c-Fos-positive and NGF-positive cells in the neuronal voiding centers [MPA, vlPAG, PMC and spinal cord (L4-L5)] were counted hemilaterally through a light microscope (BX-51; Olympus 
A

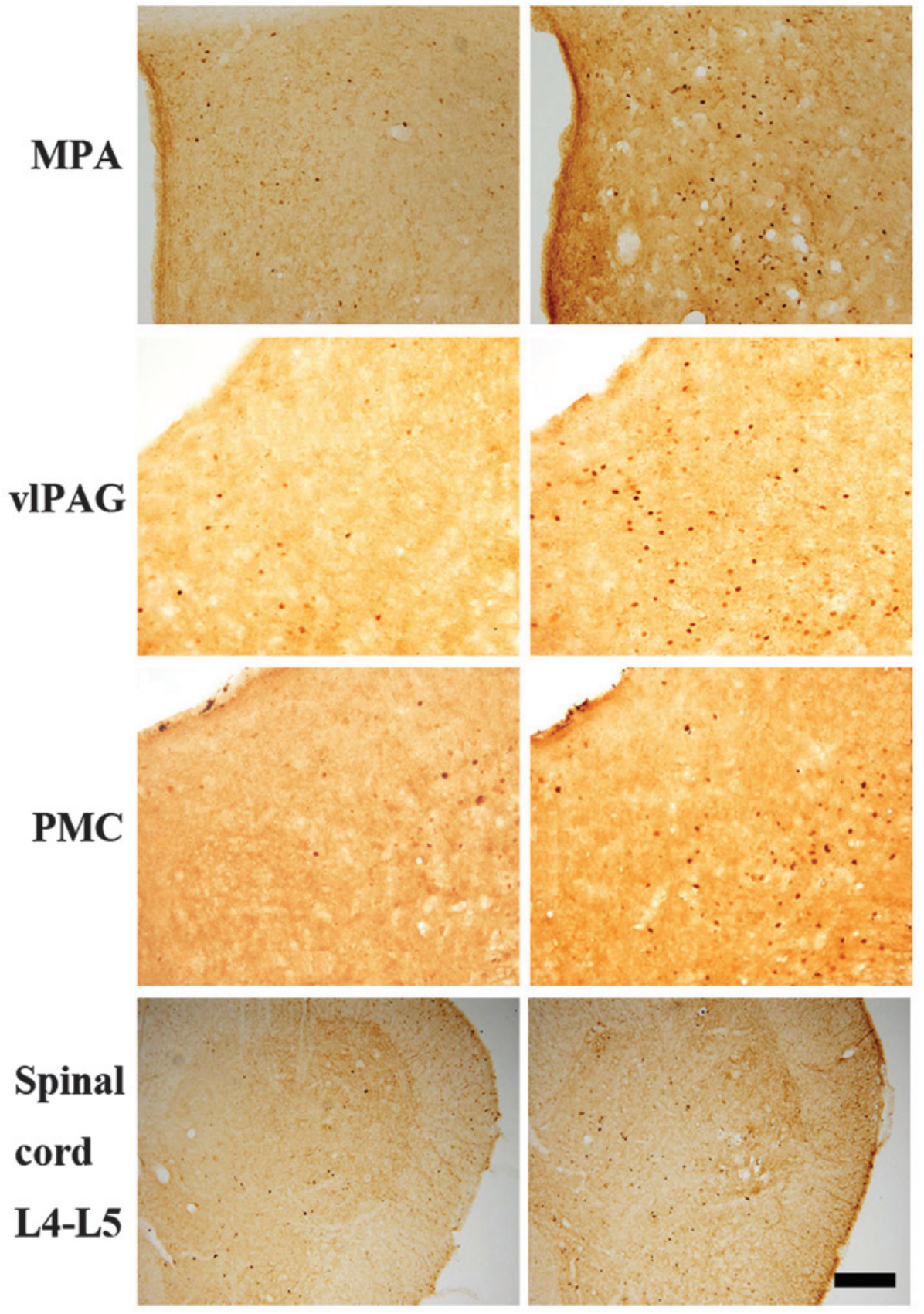

B
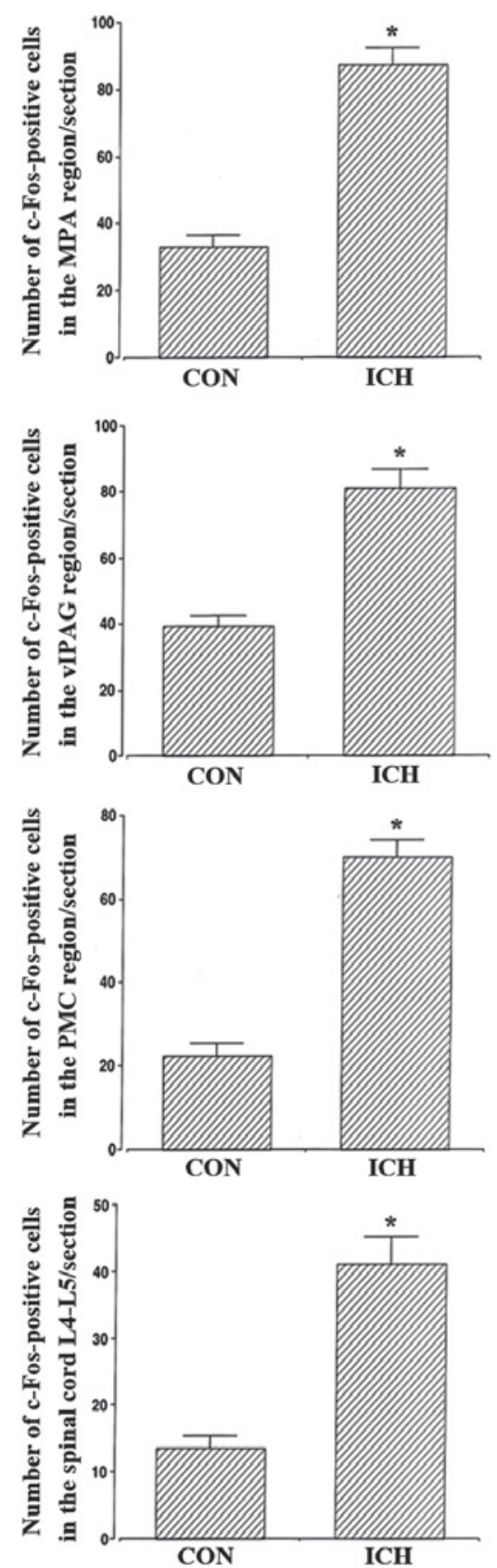

Figure 2. Effects of ICH induction on c-Fos expression in the neuronal voiding centers. (A) Photomicrographs of c-Fos-positive cells in the neuronal voiding centers. The sections were stained for c-Fos (brown staining). Scale bar, $200 \mu \mathrm{m}$. (B) Number of c-Fos-positive cells in each group. The results are presented as the mean \pm standard error of the mean. "P<0.05 vs. the control group. $\mathrm{ICH}$, intracerebral hemorrhage; CON, control group; MPA, medial preoptic area; vlPAG, ventrolateral gray; PMC, pontaine micturition center; L, lumbar.

Corporation, Tokyo, Japan). The area of the neuronal voiding center in each slice was measured using the Image-Pro ${ }^{\circledR}$ Plus computer-assisted image analysis system (Media Cyberbetics Inc., Silver Spring, MD, USA) attached to the light microscope (BX-51; Olympus Corporation). The data were analyzed using SPSS software (ver. 20.2; IBM, Armonk, NY, USA) and are expressed as the mean \pm standard error of the mean. The results of the sham surgery and $\mathrm{ICH}$-induced groups were compared using an independent paired Student's t-test. $\mathrm{P}<0.05$ was considered to indicate a statistically significant difference.

\section{Results}

Effect of ICH on urodynamic parameters. The bladder contraction and voiding parameters (pressure and time) obtained by cystometry are presented in Fig. 1 and Table I. These results indicate that bladder contraction pressure $(\mathrm{P}<0.05)$ and time $(\mathrm{P}<0.05)$ were significantly increased by the induction of $\mathrm{ICH}$ as compared with the control treatment. By contrast, the voiding pressure $(\mathrm{P}<0.05)$ and time $(\mathrm{P}<0.05)$ were significantly reduced in the rats with $\mathrm{ICH}$ induction compared with the control rats. 
A
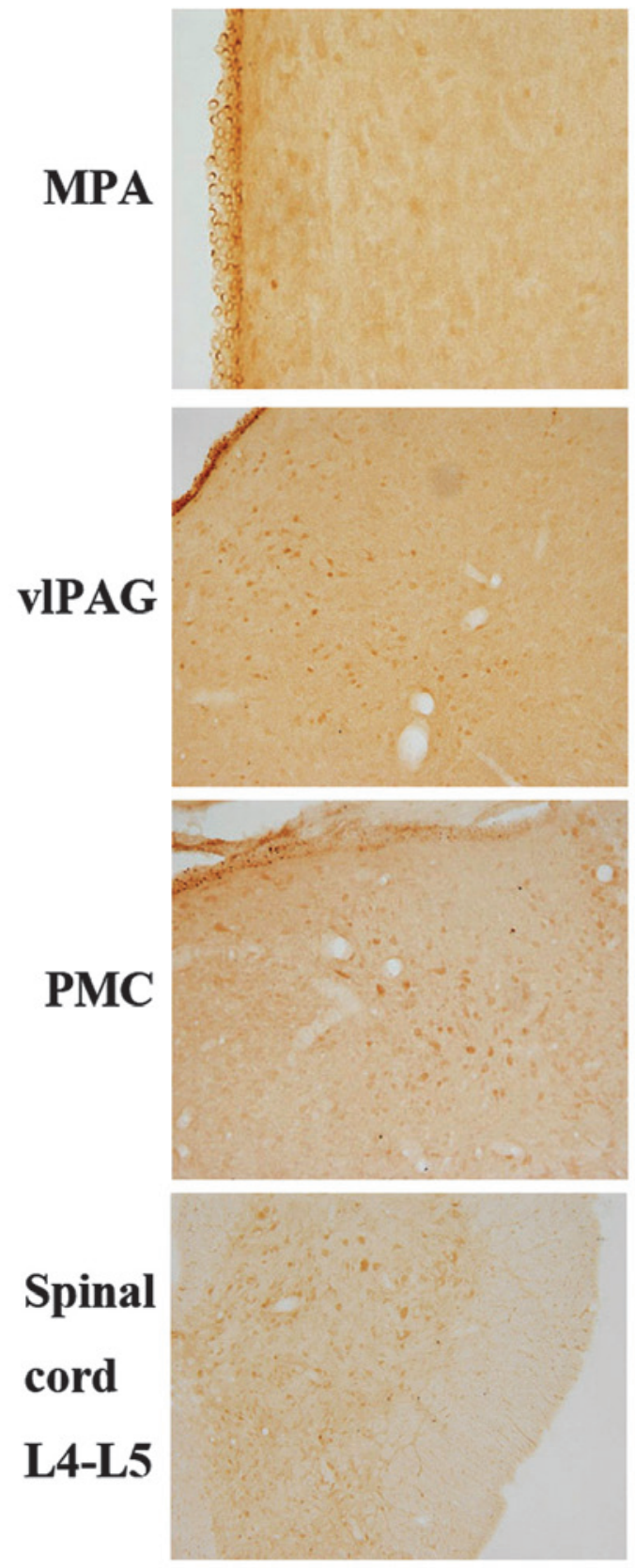

ICH
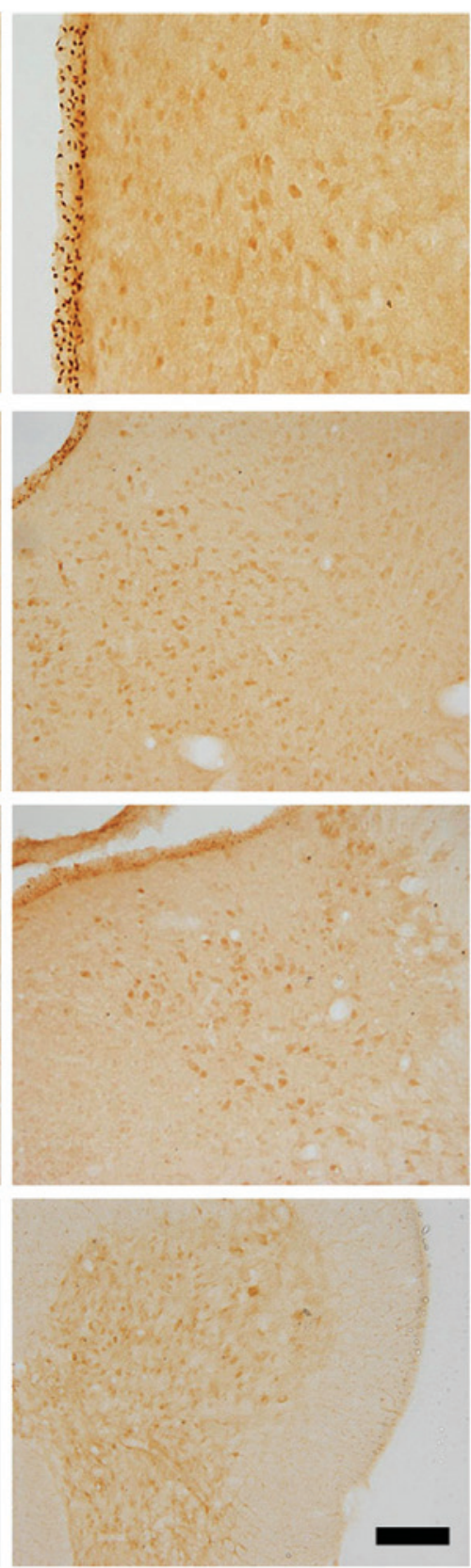

B


Figure 3. Effect of ICH induction on NGF expression in the neuronal voiding centers. (A) Photomicrographs of c-Fos-positive cells in the neuronal voiding centers. The sections were stained for c-Fos (brown staining). Scale bar, $200 \mu \mathrm{m}$. (B) Number of NGF-positive cells in each group. Results are presented as the mean \pm standard error of the mean. ${ }^{*} \mathrm{P}<0.05$ vs. the control group. ICH, intracerebral hemorrhage; NGF, nerve growth factor; CON, control group; MPA, medial preoptic area; vlPAG, ventrolateral gray; PMC, pontaine micturition center; L, lumbar.

Effect of ICH induction on c-Fos expression in the neuronal voiding centers. The results of the analysis of c-Fos-positive cells in the neuronal voiding centers are presented in Fig. 2 and Table I. These results indicate that the c-Fos expression levels in the neuronal voiding centers were significantly increased in the rats with induction of ICH compared with the control rats $(\mathrm{P}<0.05)$.

Effects of ICH induction on NGF expression in the neuronal voiding centers. The results from the analysis of NGF-positive cells in the neuronal voiding centers are presented in Fig. 3 and Table I. These results indicate that NGF expression levels in the neuronal voiding centers were significantly increased in the rats with induction of ICH compared with the control rats $(\mathrm{P}<0.05)$.

\section{Discussion}

The terms ICH and hemorrhagic stroke are used interchangeably and epidemiological studies indicate that $8-18 \%$ of strokes result from hemorrhagic insult (11). In ICH, bleeding occurs directly into the brain parenchyma. The predominant mechanism is considered to be leakage from small intracerebral arteries damaged by chronic hypertension (11). Furthermore, 
in $20-40 \%$ of patients with ischemic infarction, hemorrhagic transformation may occur within one week of ictus (12). Therefore, hemorrhage is an important pathophysiological factor in hemorrhagic and ischemic stroke.

Urinary symptoms are associated with disability and are reported to exert a considerable impact on the quality of life of stroke survivors (13). Urinary incontinence (UI) has been reported in $47 \%$ of stroke patients in the acute phase and in $19 \%$ of patients at the six-month follow-up (2). Urinary retention has been reported in $47 \%$ of stroke patients within $72 \mathrm{~h}$ of the cerebrovascular accident (1) and in $29 \%$ within four weeks of the stroke (3). Urinary symptoms, including alterations in urinary frequency, nocturia and a significantly higher rate of urinary tract infections, are observed in patients with urinary retention or incomplete bladder emptying (3). In a previous study, the frequencies of urine storage and emptying disorders in hemorrhagic and ischemic stroke patients with persistent bladder dysfunction were similar: $73.3 \%$ in the hemorrhagic stroke group versus $63.6 \%$ in the ischemic stroke group (14).

However, the majority of experimental studies of the pathophysiology and treatment of NLUTD in stroke have used a cerebral infarction animal model (4). This experimental model, however, does not accurately reflect hemorrhagic stroke. Therefore, this is not an optimal animal model in which to analyze NLUTD associated with hemorrhagic stroke. The induction of ICH by direct collagenase injection into the brain has been used in previous neurological studies $(9,10)$. In the present study, previously reported procedures for ICH induction in the hippocampal CA1 region using a stereotaxic frame and type IV collagenase were adopted, and ICH-induced changes in bladder function and neuronal voiding centers were investigated.

Bladder contraction pressure and time were found to be significantly increased in $\mathrm{ICH}$-induced rats compared with normal rats, whereas the voiding pressure and time were significantly reduced by the induction of $\mathrm{ICH}$, which indicated that $\mathrm{ICH}$ resulted in a deterioration in bladder function and as a result, the induction of OAB. Furthermore, the reductions observed in voiding pressure and time in the $\mathrm{ICH}$-induced rat model are consistent with the findings in various previous OAB models (6-8), demonstrating that OAB was induced in the ICH-induced rat model in the present study. The findings from the present study provide further support for those from a previous study that reported a significant reduction in bladder capacity $2 \mathrm{~h}$ after middle cerebral artery (MCA) occlusion in rats (15). Furthermore, the mean micturition threshold pressure increased significantly in the cerebral infarction group in that previous study, but the bladder contraction pressure was not significantly affected by left MCA occlusion compared with a sham group (15). In another study, cerebral infarction induced by MCA occlusion reduced bladder capacity in rats, but did not produce a change in bladder contraction pressure (16). The discrepancy in bladder contraction results between the present study and previous studies may be due to differences in the stroke mechanism, namely hemorrhage versus ischemia. This highlights the importance of the method used to induce stroke in experimental animal NLUTD models.

The PMC is important in the control of urinary bladder function. The hypothalamic PAG and MPA regions have been associated with the PMC (17). The PAG-PMC projection is hypothesized to be involved in the micturition reflex. The vlPAG functions as a central sensorimotor integrative relay of the micturition reflex via the reception of sensory information concerning bladder fullness and this area directly projects to the PMC (18). Neurons in the PAG regulate the micturition reflex in animals and humans, since lesions in the PAG cause severe urinary dysfunction $(18,19)$. In addition, the PMC is densely innervated by the MPA (18). The MPA sends projections to the PMC that synapse on neurons directly through projections to the spinal cord (17).

c-Fos expression occasionally serves as a marker for stimuli-induced changes in the metabolic activity of neurons under various conditions (20). In a previous study, stimulation of lower urinary tract symptoms (LUTS) was shown to cause changes in neuronal activity in neuronal micturition centers (21). Increased NGF levels have been observed in the urine of patients with interstitial cystitis and painful bladder contractions (22). NGF overexpression in the bladder and urethra is associated with modulation disability of micturition in patients with UI $(23,24)$. One biochemical study demonstrated that NGF regulates afferent bladder neuronal plasticity subsequent to partial urethral obstruction in female rats (24). Increased levels of NGF in the bladder, spinal cord and dorsal root ganglia have been associated with bladder hyperreflexia following SCI in rats (25).

In the present study, the expression levels of c-Fos and NGF in the neuronal voiding centers was significantly increased following the induction of $\mathrm{ICH}$, indicating that $\mathrm{ICH}$ facilities bladder instability through enhanced neuronal activation in central micturition areas. These findings are consistent with those of a previous study that reported increased NGF levels in the bladder tissues and urine of patients with OAB and detrusor overactivity (26), and increased c-Fos expression levels in the neuronal voiding centers in OAB model rats (27). Understanding the underlying mechanisms by which $\mathrm{ICH}$ affects urinary function is important. The limbic system is a complex set of neurological structures that lies on the two sides of the thalamus immediately under the cerebrum, and includes the hypothalamus, hippocampus, amygdala and a number of proximal areas (28). These limbic systems are known modulators of urinary function. In particular, the hypothalamus has monosynaptic connections with the PAG and PMC (28), and modulates the bladder reflex and therefore control of urinary function. Animal studies have shown connections between the thalamus and the prefrontal regions and also the PAG, which indicates that this structure is important in the relay of information, presumably including bladder sensation (21). Matsuura et al (29) noted that the thalamus was activated during the 'full bladder' state. Therefore, hemorrhagic attacks of the limbic system result in changes in the synapses of limbic areas, including the hypothalamus and hippocampus, and neuronal activity alteration in the neuronal voiding areas. NLUTD, including $\mathrm{OAB}$ and UI, may result from these alterations.

The ICH-induced NLUTD rat model in the present study may therefore be a more appropriate model to analyze NLUTD in stroke patients than the cerebral infarction model, as the model in the present study more accurately reflects the nature of the hemorrhage in hemorrhagic and ischemic stroke. Little consensus has been reached with regard to how stroke patients with NLUTD should be treated or monitored, and current treat- 
ment options for NLUTD are also limited. Animal studies are required for the examination of the efficacy or toxicity of novel medications. In the present study, ICH-induced expression of c-Fos and NGF in the neuronal voiding centers was observed in an animal model of NLUTD. Enhanced c-Fos and NGF expression levels in the voiding centers indicates activated neuronal systems that presumably induce LUTS associated with NLUTD. Thus, the animal model developed in the present study may be useful for future investigations of NLUTD associated with stroke, particularly hemorrhagic stroke.

\section{Acknowledgements}

The abstract and figures of the present study were presented as a poster during podium session 28 of the International Continence Society 2014 annual meeting, held in Rio de Janeiro, Brazil between the 20th and 24th October 2014. The present study was supported by a grant from the National Research Foundation of Korea (grant no. NRF-2012R1A1A1013173).

\section{References}

1. Burney TL, Senapati M, Desai S, Choudhary ST and Badlani GH: Acute cerebrovascular accident and lower urinary tract dysfunction: a prospective correlation of the site of brain injury with urodynamic findings. J Urol 156: 1748-1750, 1996.

2. Nakayama H, Jørgensen HS, Pedersen PM, Raaschou HO and Olsen TS: Prevalence and risk factors of incontinence after stroke. The Copenhagen Stroke Study. Stroke 28: 58-62, 1997.

3. Kong KH and Young S: Incidence and outcome of poststroke urinary retention: A prospective study. Arch Phys Med Rehabil 81: 1464-1467, 2000.

4. Fry CH, Daneshgari F, Thor K, et al: Animal models and their use in understanding lower urinary tract dysfunction. Neurourol Urodyn 29: 603-608, 2010.

5. Kajbafzadeh AM, Mohammadinejad P, Hojjat A, et al: The timing of established detrusor hyperreflexia in a rat model of neuropathic bladder. J Surg Res 178: 346-351, 2012.

6. Ozsoy O, Ozsoy U, Stein G, et al: Functional deficits and morphological changes in the neurogenic bladder match the severity of spinal cord compression. Restor Neurol Neurosci 30: 363-381, 2012

7. Kitta T, Kakizaki H, Tanaka H, et al: An alpha-amino-3-hydrox y-5-methyl-4-isoxazolepropionate glutamate-receptor antagonis can inhibit premicturition contractions in rats with bladder outlet obstruction. BJU Int 100: 181-186, 2007.

8. Juszczak K, Gil K, Wyczolkowski M and Thor PJ: Functional, histological structure and mastocytes alterations in rat urinary bladders following acute and [corrected] chronic cyclophosphamide treatment. J Physiol Pharmacol 61: 477-482, 2010.

9. Ohnishi M, Katsuki H, Fujimoto S, et al: Involvement of thrombin and mitogen-activated protein kinase pathways in hemorrhagic brain injury. Exp Neurol 206: 43-52, 2007.

10. Ohnishi M, Katsuki H, Fukutomi C, et al: HMGB1 inhibitor glycyrrhizin attenuates intracerebral hemorrhage-induced injury in rats. Neuropharmacology 61: 975-980, 2011.

11. Feigin VL, Lawes CM, Bennett DA and Anderson CS: Stroke epidemiology: a review of population-based studies of incidence, prevalence, and case-fatality in the late 20 th century. Lancet Neurol 2: 43-53, 2003.
12. Mullins ME, Lev MH, Schellingerhout D, Gonzalez RG and Schaefer PW: Intracranial Hemorrhage Complicating Acute Stroke: How Common is Hemorrhagic Stroke on Initial Head CT Scan and How Often is Initial Clinical Diagnosis of Acute Stroke Eventually Confirmed? AJNR Am J Neuroradiol 26: 2207-2212, 2005.

13. Brittain KR, Perry SI, Peet SM, et al: Prevalence and impact of urinary symptoms among community-dwelling stroke survivors. Stroke 31: 886-891, 2000.

14. Ersoz M, Tunc H, Akyuz M and Ozel S: Bladder storage and emptying disorder frequencies in hemorrhagic and ischemic stroke patients with bladder dysfunction. Cerebrovasc Dis 20: 395-399, 2005.

15. Nagasaka Y, Yokoyama O, Komatsu K, et al: Effects of opioid subtypes on detrusor overactivity in rats with cerebral infarction. Int J Urol 14: 226-232, 2007.

16. Yusup A, Akino H, Miwa Y, et al: Effects of antimuscarinics on voiding function after cerebral infarction in a rat model of overactive bladder. Eur J Pharmacol 577: 143-149, 2007.

17. Rickey LM, Sarkey S and DonCarlos LL: Estrogen-sensitive projections from the medial preoptic area to the dorsal pontine tegmentum, including Barrington's nucleus, in the rat. Neurourol Urodyn 27: 440-445, 2008.

18. Blok BF and Holstege G: Direct projections from the periaqueductal gray to the pontine micturition center (M-region). An anterograde and retrograde tracing study in the cat. Neurosci Lett 166: 93-96, 1994.

19. Sakakibara R, Hattori T, Yasuda K, et al: Micturitional disturbance in Wernicke's encephalopathy. Neurourol Urodyn 16: 111-115, 1997.

20. Dragunow M and Faull R: The use of c-fos as a metabolic marker in neuronal pathway tracing. J Neurosci Methods 29: 261-265, 1989.

21. Kavia RB, Dasgupta R and Fowler CJ: Functional imaging and the central control of the bladder. J Comp Neurol 493: 27-32, 2005.

22. Okragly AJ, Niles AL, Saban R, et al: Elevated tryptase, nerve growth factor, neurotrophin-3 and glial cell line-derived neurotrophic factor levels in the urine of interstitial cystitis and bladder cancer patients. J Urol 161: 438-442, 1999.

23. Furuta A, Kita M, Suzuki Y, et al: Association of overactive bladder and stress urinary incontinence in rats with pudendal nerve ligation injury. Am J Physiol Regul Integr Comp Physiol 294: R1510-R1516, 2008.

24. Schnegelsberg B, Sun TT, Cain G, et al: Overexpression of NGF in mouse urothelium leads to neuronal hyperinnervation, pelvic sensitivity, and changes in urinary bladder function. Am J Physiol Regul Integr Comp Physiol 298: R534-R547, 2010.

25. Seki S, Sasaki K, Fraser MO, et al: Immunoneutralization of Nerve Growth Factor in Lumbosacral Spinal Cord Reduces Bladder Hyperreflexia in Spinal Cord Injured Rats. J Urol 168: 2269-2274, 2002.

26. Liu HT, Chancellor MB and Kuo HC: Urinary Nerve Growth Factor Levels are Elevated in Patients with Detrusor Overactivity and Decreased in Responders to Detrusor Botulinum Toxin-A Injection. Eur Urol 56: 700-706, 2009.

27. Kim SE, Shin MS, Kim CJ, et al: Effects of Tamsulosin on Urinary Bladder Function and Neuronal Activity in the Voiding Centers of Rats with Cyclophosphamide-induced Overactive Bladder. Int Neurourol J 16: 13-22, 2012.

28. Holstege G: Micturition and the soul. J Comp Neurol 493: 15-20, 2005.

29. Matsuura S, Kakizaki H, Mitsui T, et al: Human brain region response to distention or cold stimulation of the bladder: a positron emission tomography study. J Urol 168: 2035-2039, 2002. 